\title{
Subclinical psychotic experiences and subsequent contact with mental health services
}

Vishal Bhavsar, James H. Maccabe, Stephani L. Hatch, Matthew Hotopf, Jane Boydell and Philip McGuire

\section{Background}

Although psychotic experiences in people without diagnosed mental health problems are associated with mental health

service use, few studies have assessed this prospectively or measured service use by real-world clinical data.

\section{Aims}

To describe and investigate the association between psychotic experiences and later mental health service use, and to assess the role of symptoms of common mental health disorders in this association.

\section{Method}

We linked a representative survey of south-east London (SELCOH-1, $n=1698$ ) with health records from the local mental healthcare provider. Cox regression estimated the association of PEs with rate of mental health service use.

\section{Results}

After adjustments, psychotic experiences were associated with a 1.75-fold increase in the rate of subsequent mental health service use (hazard ratio (HR) 1.75, 95\% Cl 1.03-2.97) compared with those without PEs. Participants with PEs experienced longer care episodes compared with those without.

\section{Conclusions}

Psychotic experiences in the general population are important predictors of public mental health need, aside from their relevance for psychoses. We found psychotic experiences to be associated with later mental health service use, after accounting for sociodemographic confounders and concurrent psychopathology.

\section{Declaration of interest}

None.

\section{Copyright and usage}

(c) The Royal College of Psychiatrists 2017. This is an open access article distributed under the terms of the Creative Commons Attribution (CC BY) license.
Psychotic experiences, such as hallucinations, thought interference and paranoid ideas, are common in the general population. However, they are only severe and/or frequent enough to reach diagnostic thresholds for schizophrenia or related psychotic disorders in a minority. ${ }^{1}$ Although people who report psychotic experiences in unselected, population-based samples have elevated risk for psychosis relative to people who do not, ${ }^{2}$ the absolute increase in this risk is low, due to the low incidence of psychotic disorders overall. ${ }^{3}$

If psychotic experiences are to be useful in predicting, and possibly averting, the later onset of clinical disorders, a key question is whether, and to what extent, these experiences are specifically associated with psychotic disorders or are associated with psychiatric disorders more generally. ${ }^{4}$ This issue is critically relevant to the current debate about whether early detection mental health services should be targeted at reducing the risk of a later psychotic disorder (e.g. in the context of the at-risk mental state (ARMS) $)^{5}$ or offer broader interventions aimed at reducing the risk of a range of mental health disorders. ${ }^{6,7}$

Although previous research has demonstrated consistent associations between psychotic experiences and use of mental health services for mental health problems such as anxiety, depression and post-traumatic stress disorder (PTSD), no studies to date have characterised this association by real-time clinical data, with objective, as opposed to self-reported, measures of service use. Furthermore, the extent to which symptoms of common mental health disorders in nonclinical individuals with psychotic experiences (such as depressive or anxiety or post-traumatic stress (PTS) symptoms) contribute to the later use of mental health services is unclear.

The first aim of this study was to quantify the association between psychotic experiences, ascertained in a representative community survey of South London, and subsequent use of local secondary mental

*Joint senior authors healthcare services ascertained directly from electronic health records, over a period of 5 years follow-up. A secondary aim was to evaluate the role of symptoms of common mental health disorders as an alternative explanation for any association. Our main hypothesis was that psychotic experiences would be associated with a higher rate of later mental health service use. We also hypothesised that this association would persist after adjusting for the influence of symptoms of depression, anxiety and PTS.

\section{Method}

\section{Sample details}

This study linked a cross-sectional community health survey with a mental healthcare provider database. The South East London Community Health Study $(\mathrm{SELCoH})^{8}$ is a representative household survey whose first wave (SELCoH-1) took place in 2008-2010. The survey used random household sampling to identify a representative sample of adults aged 16-90 years living in Lambeth and Southwark. The sampling was clustered by household, with all adults living in selected households invited to participate. Full details of the study, its sampling methods and representativeness are published. ${ }^{8}$ Among the 1698 participants surveyed, 86\% gave permission for linkage to secondary mental health records, where those records were available. SELCoH-1 participants were therefore classified into two groups, based on whether or not linked records were found. Use of mental health services both before and after SELCoH-1 interview was collected. Data from SELCoH phase 2, a survey performed in 2012-2013 based on the same sample, were used to ascertain which individuals had left the catchment area or died. ${ }^{9}$ The SELCoH-1 study received approval from the King's College London Research Ethics Committee, reference no. CREC/07/08-152. The Clinical Record Interactive Search (CRIS) data resource received ethical approval as an anonymised data 
set for secondary analyses from Oxfordshire REC C, reference no. $08 / \mathrm{H} 0606 / 71+5$.

\section{Measures}

\section{Psychotic experiences}

The Psychosis Screening Questionnaire (PSQ) ${ }^{10}$ was used to assess psychotic experiences. This is a five-item questionnaire that assesses different psychotic symptom domains experienced in the previous year. These comprise the following: hypomania, strange experiences, paranoia, hallucinations and thought disorder. Each domain contains an initial 'probe' item, which is followed by secondary questions. Because this study was focused on non-affective psychotic symptoms, responses to the hypomania item were not examined. Individuals were considered to have psychotic experiences if they endorsed one or more secondary items in the four remaining domains. The Psychosis Screening Questionnaire displays good correspondence with psychosis items on the Schedules for Clinical Assessment in Neuropsychiatry ${ }^{10}$ and has seen frequent use in population studies. ${ }^{11}$

\section{Covariates}

SELCoH-1 collected sociodemographic, environmental and health information. For this analysis, age was grouped into 10-year categories. Ethnicity was operationalised as a five-category variable comprising White, Black African, Black Caribbean, Asian and other groups. Participants' employment status was categorised into employed, student, unemployed and other. Highest educational attainment was categorised into 'no qualifications', 'GCSE/ O-level', 'A-Level' and 'degree level and above'. PTS symptoms were assessed by the PC-PTSD, ${ }^{12}$ a screening tool for PTSD designed for primary care use, which is based on the diagnostic criteria for PTSD in DSM-IV. The Clinical Interview Schedule (Revised, CIS-R) ${ }^{13}$ was used to measure symptoms of depressive and anxiety disorders in the form of a numerical score.

\section{Use of mental health services}

Data on mental healthcare utilisation for SELCoH-1 participants were derived from the National Institute of Health Research Biomedical Research Centre at the Maudsley's Clinical Record Interactive Search. ${ }^{14}$ The South London and Maudsley NHS Foundation Trust (SLaM) is the sole provider of mental healthcare in the two boroughs of South London that were surveyed in SELCoH-1, covering a catchment population of approximately 0.62 million. The Trust has used a single electronic health record across all clinical services known as the electronic Patient Journey System (ePJS) since 2006, with more limited information available from 2001. CRIS extracts de-identified clinical data from the ePJS including structured fields for ICD-10 diagnoses, treatments and admissions to hospital. Linkage of SELCoH-1 to CRIS was carried by an independent in-house informatics team, the SLaM Clinical Data Linkage Service (CDLS), and used personal identifiers (name, date of birth, NHS number, postcode and gender) to probabilistically link survey data with matching electronic health records. ${ }^{15}$ Data on SELCoH-1 participants who had consented to record linkage were then scrutinised in CRIS. Information was available on date and route of referral to mental health services and ICD-10 diagnosis. Diagnoses in SLaM are generally arrived at through multidisciplinary discussion among the team primarily responsible for the patient. ${ }^{14}$ Primary diagnoses were grouped into common mental disorders, serious mental illness, other diagnoses and patients in whom no diagnosis was made. Durations of separate care episodes for each individual were added together to derive a variable reflecting total duration of mental health service use (grouped into total care episodes of durations between 0 and
100 days, between 101 and 1000 days, and 1001 days or above). A categorical variable on referral source contained groups for referral via accident and emergency departments, self-referral/referral from school/referral from a carer, referral by a general practitioner, a non-psychiatric clinical specialty, police/medium secure mental health services, social services, other services and a category for participants where the source of referral was missing or recorded as null.

\section{Analysis}

All analyses were carried out in STATA $14^{16}$ and took account of household non-response and clustering of respondents within households, by inverse probability weights and survey commands in STATA. The estimation of non-response weights is further described in Hatch et al. ${ }^{8}$ Counts/percentages for categorical variables were described for those who made contact with mental health services and those who did not. Categorical distributions for linked participants, for diagnosis, care duration and referral source were described by psychotic experience status and evaluated by chi-squared tests. For Cox regression, only use of mental health services commencing after the survey interview was analysed; time was defined as elapsed time since birth and observation period as time elapsed from SELCoH-1 interview. Conclusion of follow-up time was defined as removal from the population at risk, either due to the occurrence of the outcome (use of mental health services), due to coming to the end of follow-up time (15 May 2015) or due to being untraceable or dead at date of follow-up interview. Rates stratified by psychotic experience status, age, gender and the other sociodemographic and clinical variables were calculated. Proportionality of hazards by psychotic experience status was assessed graphically by log-log plots and a test for the null hypothesis of a zero slope in the log hazard ratio (HR) function, by Schoenfeld residuals. Covariates for Cox regression were evaluated for inclusion based on the change in estimates method - covariates for which the adjusted association changed by more than $10 \%$ from the crude association were retained for inclusion in the fully adjusted model. ${ }^{17}$ Partially and fully adjusted rate ratios were estimated for secondary mental health service use.

\section{Results}

\section{Categorical description of linkage and linked participants}

Of 1698 survey participants, 243 individuals did not consent to linkage: information on whether or not these individuals used mental healthcare was therefore not available. These records were dropped from the analysis. Chi-squared comparison of those who did with those who did not consent to linkage indicated that there were no significant differences in gender, educational attainment or employment status between the two groups. However, consenting participants were more likely to be older and of White ethnicity (data available on request from authors). The overall proportion of individuals using secondary mental health services at any time was $12.10 \%$. Nearly $40 \%$ of people who used secondary mental health services reported psychotic experiences at baseline compared with around $17 \%$ of people who did not use these services (Table 1). Of those using secondary mental health services, $64 \%$ were female compared with $55 \%$ of those who did not. Participants using secondary mental health services had lower educational attainment, were more likely to be unemployed, and were more likely to report symptoms of common mental health disorders. 
Table 1 Counts (column percentages in brackets) and crude rates of mental health service use for psychotic experiences and selected SELCOH-1 variables

\begin{tabular}{|c|c|c|c|c|}
\hline & $\begin{array}{l}\text { Did not use secondary } \\
\text { mental health services }\end{array}$ & $\begin{array}{l}\text { Used secondary } \\
\text { mental health services }\end{array}$ & $\begin{array}{l}\text { Rate (contacts per } \\
1000 \text { person-years) }\end{array}$ & $95 \% \mathrm{Cl}$ \\
\hline \multicolumn{5}{|c|}{ Psychotic experiences } \\
\hline No & 1060 (82.88) & $108(61.36)$ & 9.58 & 7.42 to 12.56 \\
\hline Yes & $214(16.73)$ & $67(38.07)$ & 19.48 & 13.14 to 30.00 \\
\hline Missing & $5(0.39)$ & $1(0.57)$ & & \\
\hline \multicolumn{5}{|l|}{ Gender } \\
\hline Male & $570(44.57)$ & $63(35.80)$ & 7.97 & 5.43 to 12.18 \\
\hline Female & 709 (55.43) & $113(64.20)$ & 14.25 & 11.04 to 19.2 \\
\hline Missing & $0(0.00)$ & $0(0.00)$ & & \\
\hline \multicolumn{5}{|l|}{ Age } \\
\hline 16-24 & 269 (21.03) & $31(17.61)$ & 11.02 & 6.81 to 18.94 \\
\hline $25-34$ & $296(23.14)$ & $26(14.77)$ & 5.67 & 2.99 to 12.11 \\
\hline $35-44$ & $264(20.64)$ & $32(18.18)$ & 9.58 & 5.74 to 17.16 \\
\hline $45-54$ & $198(15.48)$ & $36(20.45)$ & 8.92 & 4.87 to 18.08 \\
\hline $55-64$ & $126(9.85)$ & $23(13.07)$ & 14.70 & 8.31 to 28.43 \\
\hline $65+$ & $126(9.85)$ & $28(15.91)$ & 29.51 & 19.57 to 46.27 \\
\hline Missing & $0(0.0)$ & $0(0.0)$ & & \\
\hline \multicolumn{5}{|l|}{ Employment } \\
\hline Employed & 743 (58.09) & $41(23.30)$ & 5.71 & 3.83 to 8.90 \\
\hline Students & 187 (14.62) & $22(12.50)$ & 10.67 & 5.98 to 20.86 \\
\hline Unemployed & $107(8.37)$ & 37 (21.02) & 23.12 & 13.71 to 41.64 \\
\hline Other & 237 (18.53) & $75(42.61)$ & 23.52 & 16.83 to 33.76 \\
\hline Missing & $5(0.39)$ & $1(0.57)$ & & \\
\hline \multicolumn{5}{|l|}{ Education } \\
\hline No qualifications & $153(11.96)$ & $43(24.43)$ & 25.07 & 16.77 to 38.98 \\
\hline GCSE & 247 (19.31) & $52(29.55)$ & 14.16 & 9.22 to 22.82 \\
\hline A-level & 317 (24.78) & $37(21.02)$ & 10.88 & 6.99 to 17.83 \\
\hline Degree level & $553(43.24)$ & 38 (21.59) & 5.94 & 3.79 to 9.86 \\
\hline Missing & $9(0.70)$ & $6(3.41)$ & & \\
\hline \multicolumn{5}{|l|}{ Ethnicity } \\
\hline White & $814(63.64)$ & $120(68.18)$ & 11.52 & 8.88 to 15.2 \\
\hline Black Caribbean & $98(7.66)$ & $18(10.23)$ & 14.41 & 7.24 to 32.59 \\
\hline Black African & $174(13.60)$ & $13(7.39)$ & 5.93 & 2.49 to 17.79 \\
\hline Asian & $43(3.36)$ & $5(2.84)$ & 11.70 & 3.61 to 56.60 \\
\hline Other & $148(11.57)$ & $20(11.36)$ & 13.45 & 7.56 to 26.17 \\
\hline Missing & $2(0.16)$ & $0(0.00)$ & & \\
\hline \multicolumn{5}{|c|}{ Post-traumatic stress symptoms } \\
\hline None & $1219(95.31)$ & $147(83.52)$ & 10.47 & 8.34 to 13.31 \\
\hline Possible case & $48(3.75)$ & 27 (15.34) & 29.89 & 15.21 to 66.03 \\
\hline Missing & $12(0.94)$ & $2(1.14)$ & & \\
\hline \multicolumn{5}{|c|}{ Symptoms of common mental health disorder } \\
\hline No & $1010(78.97)$ & $92(52.27)$ & 7.79 & 5.84 to 10.61 \\
\hline Yes & $268(20.95)$ & $82(46.59)$ & 23.79 & 17.18 to 33.77 \\
\hline Missing & $1(0.08)$ & $2(1.14)$ & & \\
\hline Total & $1279(100)$ & $176(100)$ & 11.24 & 9.07 to 14.10 \\
\hline
\end{tabular}

\section{Use of mental health services}

In participants with any mental health service use, psychotic experiences were not statistically associated with either diagnoses or referral source. However, a greater proportion of those who reported psychotic experiences had care episodes totalling 1000 days or more in duration, compared with those who did not report psychotic experiences (Table 2). Among 15 individuals who used mental health services who were assigned the diagnostic grouping of serious mental illness, 12 were recorded as having psychotic disorders and 3 were diagnosed with bipolar affective disorder.

\section{Survival analysis of time to first mental health service use}

Description of survival data

Of 1455 survey participants consenting to linkage of their survey data with electronic health records, person-time for 94 individuals was excluded as follow-up had concluded before the baseline assessment interview (i.e. they used mental health services before the SELCoH-1 interview). Remaining were 1361 participants, among whom there were 82 individuals who used mental health services after interview. Of the remaining participants, 12 died after the survey interview, and 105 were found to be untraceable at follow-up interview and considered to have been censored at the date that contact was attempted. The total follow-up time in the whole sample was 6926.28 years. Per individual, the median follow-up time was 5.25 years. Among participants without psychotic experiences at survey interview, median followup time was 5.27 years, and in those with psychotic experiences, it was 5.20 years. We found no statistical evidence for a difference in follow-up times between those with and without psychotic experiences, based on a Wilcoxon rank-sum test $(P=0.522)$. Followup time did not vary significantly by gender or other sociodemographic variables analysed in this study (data available on request from authors). 
Table 2 Service use information on SELCOH-1 participants who used secondary mental healthcare, by psychotic experience (PE) status $(n=175)$

Chi-squared No PES (\%) PES (\%) P-value (d.f.)

$\begin{array}{lcc}\text { Diagnostic group } & & \\ \text { CMD } & 37(34.26) & 28(41.79) \\ \text { SMl } & 8(7.41) & 7(10.45) \\ \text { Dementia } & 9(8.33) & 2(2.99) \\ \text { Other } & 18(16.67) & 8(11.94) \\ \text { Null } & 36(33.33) & 22(32.84)\end{array}$

Total duration of contact within

mental healthcare

Less than 100 days $\quad 49(45.37) \quad 20(29.85)$

100 to less than 1000 days $\quad 45(41.67) \quad 29(43.28)$

1000 days or more $\quad 14(12.96) \quad 18(26.87)$

Referral source

Emergency department $\quad 11(10.19) \quad 9$ (13.43)

Self/school/carer $\quad 5(4.63) \quad 5(7.46)$

GP 38 (35.19) 25 (37.31)

Null $\quad 8$ (7.41) 1 (1.49)

Other $25(23.15) \quad 20(29.85)$

Other specialty $\quad 16(14.81) \quad 6(8.96)$

Police/MSU 1 (0.93) 1 (1.49)

Social services $\quad 4(3.70) \quad 0(0.0)$

Total $108(100) \quad 67(100)$

CMD, Common mental disorders; SMl, severe mental illness; MSU, medium secure unit.

The overall rate of mental health service use in the sample population was around 11 contacts per 1000 person-years of followup (rate $=11.24,95 \%$ CI 9.07 to 14.10 ; Table 1). People who reported psychotic experiences at survey interview had around twice the crude rate of subsequent mental health service use compared with those who did not. A higher rate of mental health service use was associated with female gender, lower educational attainment, unemployed status and baseline symptoms of depression, anxiety and PTSD (Table 1).

\section{Cox regression analysis}

Among the 1361 participant records providing follow-up information, 24 individuals had missing data on one or more of the modelled variables and were removed from the analysis, leaving a final analytic sample for multivariate modelling of 1337. Graphical and inferential methods did not indicate that proportional hazards assumptions had been violated ( $P$-value from the formal test of the proportional hazards assumption, based on Schoenfeld residuals, was 0.5164 , see Fig. 1). In multivariate Cox regression, psychotic experiences were associated with a twofold increase in the rate of mental health service use, after adjusting for age, gender, employment status, educational attainment and ethnic group ( $\mathrm{HR}=1.91$, $95 \%$ CI 1.13 to 3.26 ). This association was partially attenuated on inclusion of symptoms of depression and anxiety, and this association was further reduced by the addition of PTS symptoms. Nevertheless, good statistical evidence remained for an association between reporting psychotic experiences and time to mental health service use, after accounting for these variables ( $\mathrm{HR}=1.75,95 \% \mathrm{CI}$ 1.03 to 2.97 ; Table 3 ).

\section{Discussion}

\section{Summary of findings}

This investigation found that in a representative population sample, psychotic experiences were prospectively associated with approximately a doubling in the likelihood that they would use secondary mental health services over the next 5 years. This effect was partially, but not completely, accounted for by concurrent symptoms of depression, anxiety and PTS at baseline. Survey participants who had reported psychotic experiences spent longer under the care of mental health services than those who had not reported psychotic experiences.

\section{What is already known on this topic}

Psychotic experiences are distressing, ${ }^{18}$ and a number of studies have reported an association between psychotic experiences and

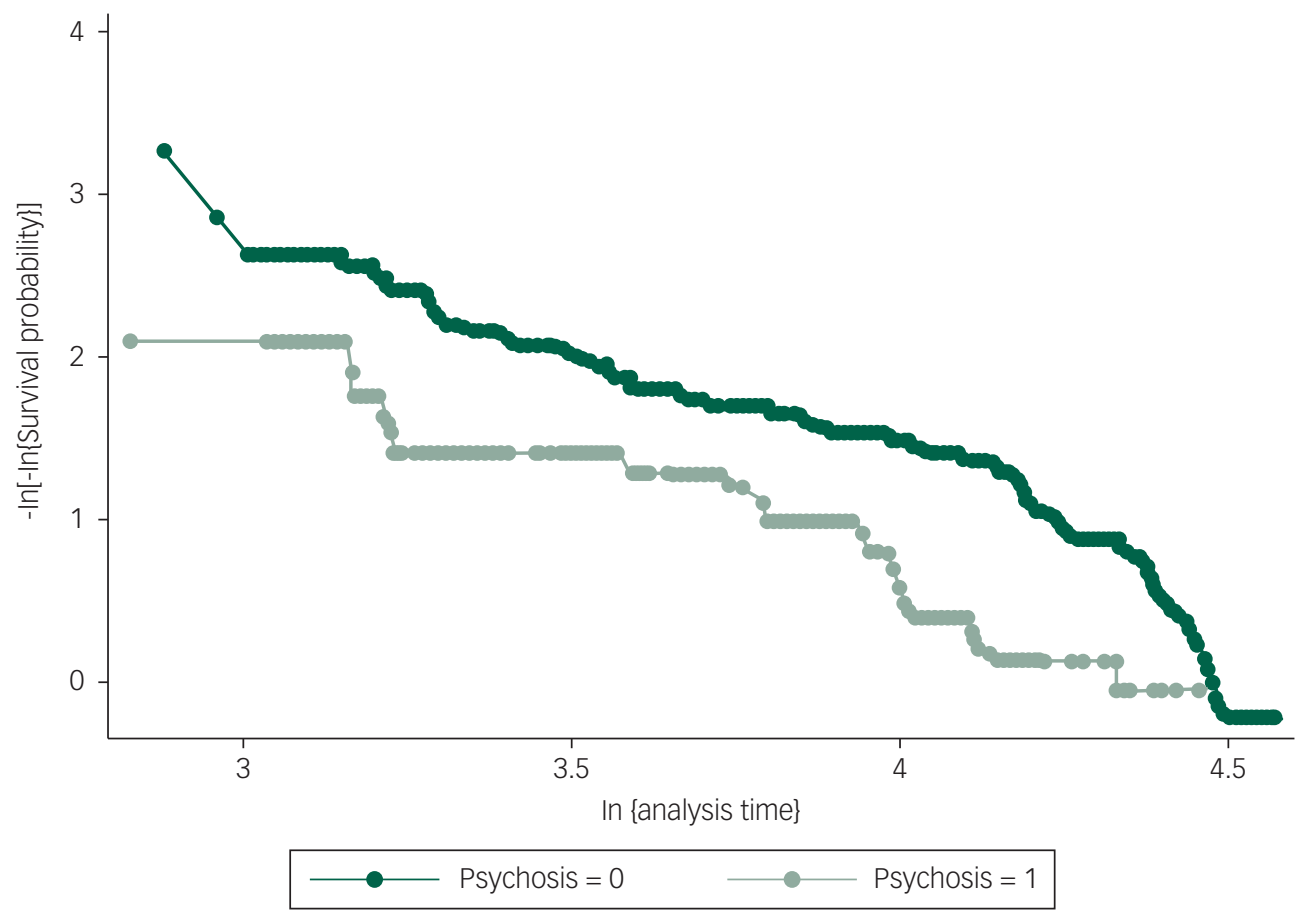

Fig. 1 Approximately parallel curves for psychotic experiences and no psychotic experiences on axes of -log[log(survival probability)] against log (analysis time), implying that proportionality of hazards has not been violated. Global test of proportional hazards assumption for the final regression model, based on Schoenfeld residuals, was 0.516 
Table 3 Multivariate Cox regression models presenting hazard ratios (HRs) for the effect of psychotic experiences on the hazard of contact with mental health services

\begin{tabular}{|c|c|c|c|}
\hline & $\begin{array}{c}\text { HR comparing psychotic experiences with } \\
\text { no psychotic experiences }\end{array}$ & $95 \% \mathrm{Cl}$ & $P$ \\
\hline Adjusted for age only & 2.41 & 1.48 to 3.92 & $<0.001$ \\
\hline Adjusted for age and gender & 2.39 & 1.46 to 3.92 & 0.001 \\
\hline Adjusted for age, gender and educational attainment & 2.13 & 1.28 to 3.55 & 0.004 \\
\hline Adjusted for age, gender, educational attainment and employment & 1.87 & 1.11 to 3.17 & 0.02 \\
\hline $\begin{array}{l}\text { Adjusted for age, gender, educational attainment, employment and ethnic } \\
\text { group }\end{array}$ & 1.91 & 1.13 to 3.26 & 0.016 \\
\hline $\begin{array}{l}\text { Adjusted for age, gender, educational attainment, employment, ethnic } \\
\text { group and symptoms of common mental health disorders }\end{array}$ & 1.90 & 1.12 to 3.22 & 0.017 \\
\hline $\begin{array}{l}\text { Adjusted for age, gender, educational attainment, employment, ethnic } \\
\text { group, symptoms of common mental health disorders and PTS symptoms }\end{array}$ & 1.75 & 1.03 to 2.97 & 0.039 \\
\hline
\end{tabular}

use of mental health services, in both community ${ }^{19}$ and clinical high-risk samples. ${ }^{6}$ One study did not find an association ${ }^{20}-$ this study investigated a psychiatric out-patient group, comparing those who returned following an initial assessment with those who did not.

Previous studies examining service use in individuals reporting psychotic experiences have not measured service use subsequent to their assessment or attempted to estimate the effect of psychotic experiences on service use duration. Armando et $a l^{21}$ reported a strong correlation between subtypes of psychotic experiences and use of mental health services, but it is unclear what proportion of people with any psychotic experiences reported service use. Devylder et $a^{22}$ analysed data from the Collaborative Psychiatric Epidemiology Survey to investigate the use of mental health services in people with and without psychotic experiences and found that nearly $60 \%$ of people with psychotic experiences reported lifetime mental health service use, compared with around $40 \%$ of people without. In an analysis of the Adult Psychiatric Morbidity survey 2007, Murphy et al ${ }^{19}$ reported elevated risks of lifetime use of mental health services in people with psychotic experiences, with paranoid experiences associated with a more than twofold increase in risk of reporting contact with mental health services in the previous year. However, all of these studies relied on self-reported service use, ${ }^{19,21-23}$ which could be vulnerable to recall bias; ${ }^{24}$ for example, individuals who have more extensive mental health service use and may have the most severe disorders, or may have better recall of service use than individuals with more transient distress. ${ }^{25}$

Overall, the previous literature suggests that psychotic experiences are associated with an increased likelihood of using mental health services, with more limited evidence that this association remains after accounting for possible confounding effects of other mental health symptoms. However, the assessment of mental health service use has usually relied on self-report rather than directly measured mental health service use, and no studies to our knowledge have assessed the association between psychotic experiences and mental health service use prospectively.

\section{What this study adds}

Our findings extend this literature by suggesting that psychotic experiences significantly increase the likelihood of subsequent use of mental health services. Moreover, this association was evident in a prospective follow-up of a large sample gathered via a representative household survey. Service use was measured by directly analysing mental health records from the comprehensive mental healthcare provider for the local population, and the association was not simply attributable to the influence of concurrent symptoms of common mental health disorders.

\section{Explanations for the association}

Psychotic experiences could be associated with subsequent service use because they are concurrent with other mental health symptoms or conditions. Associations have been documented between psychotic experiences and symptoms of depression, ${ }^{18,26}$ anxiety, ${ }^{27,28}$ obsessive-compulsive disorder, ${ }^{29}$ PTSD, ${ }^{30,31}$ as well as suicidal behaviours. $^{32,33}$ However, we found that the effect of psychotic experiences on subsequent service use persisted after adjusting for the presence of depression, anxiety and PTS symptoms.

\section{Importance}

Our results suggest that psychotic experiences in the general population are a significant predictor of a need for later mental health service use, after accounting for the correlation between psychotic experiences and symptoms of common mental health disorders. At present, clinical care for psychotic experiences is limited to the subgroup of people who have psychotic experiences that occur as part of an at-risk mental state. ${ }^{5}$ Moreover, these services are focused on reducing the risk of psychotic disorders, as opposed to mental disorders in general. Our findings indicate that the presence of psychotic experiences is associated with an increase in later mental health service use at the level of the general population. This service use may involve a range of mental disorders rather than being limited to psychosis. ${ }^{4,7}$

\section{Strengths and limitations}

One limitation of the study was that we did not have information about how long psychotic experiences had been present before the baseline assessment, which precluded analysis of the time between the onset of psychotic experiences and subsequent use of mental health services. In addition, diagnostic information on those who used mental health services was incomplete and based on routine clinical recordings, rather than validated diagnostic measures. Although the outcome was defined on the basis of routine clinical data, thereby limiting comparison with formal epidemiological designs, the results indicate that psychotic experiences are related to subsequent mental healthcare need at the population level, irrespective of diagnosis. In this analysis, concurrent mental health symptoms were adjusted for as potential confounders. However, the relationship between different mental health symptoms and psychotic experiences could be more complex. For example, it was not possible to determine whether depression/anxiety symptoms are confounders of the association between psychotic experiences and service use, or whether they are mediators. Finally, this analysis made the assumption that those participants who did not make contact with mental health services and who were not found to be untraceable at follow-up interview remained at risk for the outcome over the duration of the study. We may therefore have overestimated the length of time people were at risk for the outcome in 
this study, underestimating the overall rate of mental health service use. However, we consider this to be a conservative measure and that any bias introduced by this was probably in the null direction. Furthermore, people moving out of the study area might be expected to be more resilient/resourceful/mobile and therefore be less likely to seek mental healthcare in the context of psychotic experiences compared with those without psychotic experiences; however, we feel this is unlikely as a mechanism of bias in this study - we expect that most participants moved out of the study area because they were displaced by urban development and regeneration and not because of outward migration of more resilient participants.

\section{Concluding remarks}

In general population-based sample of south-east London, psychotic experiences were associated with later use of mental health services over a period of 5 years of follow-up. This subsequent mental health service use was related to mental health problems in general, rather than specifically to psychotic disorders.

Vishal Bhavsar, MRCPsych, Department of Psychosis Studies, Institute of Psychiatry, Psychology and Neuroscience, King's College London, London, UK; James H. Maccabe PhD, Department of Psychosis Studies, Institute of Psychiatry, Psychology and Neuroscience, King's College London, London, UK; Stephani L. Hatch, PhD, Department of Psychological Medicine, Institute of Psychiatry, Psychology and Neuroscience, King's College London, London, UK; South London and Maudsley NHS Foundation Trust, London, UK; Matthew Hotopf, PhD, Department of Psychological Medicine, Institute of Psychiatry, Psychology and Neuroscience, King's College London, London, UK; South London and Maudsley NHS Foundation Trust, London, UK; Jane Boydell, PhD, Cornwall Partnership NHS Foundation Trust, Bodmin, UK Philip McGuire, PhD, Department of Psychosis Studies, Institute of Psychiatry, Psychology and Neuroscience, King's College London, London, UK

Correspondence: Vishal Bhavsar, Department of Psychosis Studies, Institute of Psychiatry, Psychology and Neuroscience, King's College London, De Crespigny Park London SE5 8AF, UK. Email: vishal.2.bhavsar@kcl.ac.uk

First received 3 Feb 2017, final revision 8 Feb 2017, accepted 8 Feb 2017

\section{Acknowledgements}

The authors wish to acknowledge the assistance of Craig Morgan, Robert Stewart and David Pernet in this research.

\section{Funding}

This study represents independent research funded by the National Institute for Health Research (NIHR) Biomedical Research Centre at South London and Maudsley NHS Foundation Trust and King's College London. The views expressed are those of the author(s) and not necessarily those of the NHS, the NIHR or the Department of Health. The funder had no role in the study design, in the collection, analysis and interpretation of data, in the writing of the report and in the decision to submit the article for publication. V.B. is supported by a Wellcome Trust Clinical Research Training Fellowship 101681/Z/13/Z. M.H., S.L.H. and P.M. are supported by the National Institute for Health Research (NIHR) Biomedical Research Centre at South London and Maudsley NHS Foundation Trust and King's College London.

\section{References}

1 van OS J, Linscott RJ. Introduction: the extended psychosis phenotype - relationship with schizophrenia and with ultrahigh risk status for psychosis. Schizophr Bul 2012; 38: 227-30.

2 Kaymaz N, Drukker M, Lieb R, Wittchen HU, Werbeloff $N$, Weiser M, et al. Do subthreshold psychotic experiences predict clinical outcomes in unselected nonhelp-seeking population-based samples? A systematic review and meta-analysis, enriched with new results. Psychol Med 2012; 42: 2239-53.

3 van Os J, Murray RM. Can we identify and treat 'schizophrenia light' to prevent true psychotic illness. BMJ 2013; 346: f304.

4 McGorry PD, Killackey E, Yung A. Early intervention in psychosis: concepts, evidence and future directions. World Psychiatry 2008; 7: 148-56.

5 Fusar-Poli P, Borgwardt S, Bechdolf A, Addington J, Riecher-Rössler A, SchultzeLutter $F$, et al. The psychosis high-risk state: a comprehensive state-of-the-art review. JAMA PSychiatry 2013; 70: 107-20.
6 Lin A, Wood SJ, Nelson B, Beavan A, McGorry P, Yung AR. Outcomes of nontransitioned cases in a sample at ultra-high risk for psychosis. Am J Psychiatry 2015; 172: 249-58.

7 Fusar-Poli P, Yung AR, McGorry P, van Os J. Lessons learned from the psychosis high-risk state: towards a general staging model of prodromal intervention. Psychol Med 2014; 44: 17-24.

8 Hatch SL, Frissa S, Verdecchia M, Stewart R, Fear NT, Reichenberg A, et al. Identifying socio-demographic and socioeconomic determinants of health inequalities in a diverse London community: the South East London Community Health (SELCOH) study. BMC Public Health 2011; 11: 861.

9 Hatch S, Gazard B, Williams D, Frissa S, Goodwin L, SELCOH Study Team, et al. Discrimination and common mental disorder among migrant and ethnic groups: findings from a South East London Community sample. Soc Psychiatry Psychiatr Epidemiol 2016; 51: 689-701.

10 Bebbington $\mathrm{P}$, Nayani T. The psychosis screening questionnaire. Int J Methods Psychiatr Res 1995; 5: 11-9.

11 Bebbington $P E$, Bhugra $D$, Brugha $T$, Singleton $N$, Farrell $M$, Jenkins $R$, et al. Psychosis, victimisation and childhood disadvantage: evidence from the second British National Survey of Psychiatric Morbidity. Br J Psychiatry 2004; 185: 220-6.

12 Prins A, Ouimette P, Kimerling R, Cameron RP, Hugelshofer DS, Shaw-Hegwer J, et al. The primary care PTSD screen (PC-PTSD): development and operating characteristics. Primary Care Psychiatry 2003; 9: 9-14.

13 Lewis G, Pelosi AJ, Araya R, Dunn G. Measuring psychiatric disorder in the community: a standardized assessment for use by lay interviewers. Psychol Med 1992; 22: 465-86.

14 Stewart R, Soremekun M, Perera G, Broadbent M, Callard F, Denis M, et al. The South London and Maudsley NHS foundation trust biomedical research centre (SLAM BRC) case register: development and descriptive data. BMC Psychiatry 2009; 9: 51.

15 Health and Social Care Information Centre. Monthly Patient Reported Outcome Measures (PROMS) in England, 2015.

16 StataCorp. Stata Statistical Software: Release 14. Special Edition. StataCorp, 2014.

17 Greenland S, Pearce N. Statistical foundations for model-based adjustments. Annual Rev Public Health 2015; 36: 89-108.

18 Armando M, Nelson B, Yung AR, Ross M, Birchwood M, Girardi P, et al. Psychoticlike experiences and correlation with distress and depressive symptoms in a community sample of adolescents and young adults. Schizophr Res 2010; 119: $258-65$

19 Murphy J, Shevlin M, Houston J, Adamson G. A population based analysis of subclinical psychosis and help-seeking behavior. Schizophr Bull 2012; 38: 360-7.

20 Kobayashi $\mathrm{H}$, Nemoto $\mathrm{T}$, Murakami M, Kashima $\mathrm{H}$, Mizuno M. Lack of association between psychosis-like experiences and seeking help from professionals: a case-controlled study. Schizophr Res 2011; 132: 208-12.

21 Armando $M$, Nelson B, Yung AR, Saba R, Monducci E, Dario C, et al. Psychotic experience subtypes, poor mental health status and help-seeking behaviour in a community sample of young adults. Early Interv Psychiatry 2012; 6: 300-8.

22 Devylder JE, Oh HY, Corcoran CM, Lukens EP. Treatment seeking and unmet need for care among persons reporting psychosis-like experiences. Psychiatr Serv 2014; 65: 774-80

23 van Nierop M, van Os J, Gunther N, Myin-Germeys I, de Graaf R, ten Have M, et al. Phenotypically continuous with clinical psychosis, discontinuous in need for care: evidence for an extended psychosis phenotype. Schizophr Bull 2012; 38: 231-8.

24 Rhodes AE, Fung K. Self-reported use of mental health services versus administrative records: care to recall? Int J Methods Psychiatr Res 2004; 13: 165-75.

25 Rhodes $A E$, Lin $E$, Mustard $C A$. Self-reported use of mental health services versus administrative records: should we care? Int I Methods Psychiatr Res 2002; 11: $125-33$.

26 Wigman JT, van Nierop M, Vollebergh WA, Lieb R, Beesdo-Baum K, Wittchen HU, et al. Evidence that psychotic symptoms are prevalent in disorders of anxiety and depression, impacting on illness onset, risk, and severity - implications for diagnosis and ultra-high risk research. Schizophr Bull 2012; 38: 247-57.

27 Fusar-Poli P, Nelson B, Valmaggia L, Yung AR, McGuire PK. Comorbid depressive and anxiety disorders in 509 individuals with an at-risk mental state: impact on psychopathology and transition to psychosis. Schizophr Bull 2014; 40: $120-31$.

28 Saha S, Scott J, Varghese D, McGrath J. Anxiety and depressive disorders are associated with delusional-like experiences: a replication study based on a National Survey of Mental Health and Wellbeing. BMJ Open 2012; 2: e001001.

29 Devylder JE, Oh AJ, Ben-David S, Azimov N, Harkavy-Friedman JM, Corcoran CM Obsessive compulsive symptoms in individuals at clinical risk for psychosis: association with depressive symptoms and suicidal ideation. Schizophr Res 2012: 140: $110-3$ 
30 Campbell ML, Morrison AP. The psychological consequences of combat exposure: the importance of appraisals and post-traumatic stress disorder symptomatology in the occurrence of delusional-like ideas. Br J Clin Psychol 2007; 46: 187-201.

31 Scott J, Chant D, Andrews G, Martin G, McGrath J. Association between trauma exposure and delusional experiences in a large community-based sample. $\mathrm{Br} J$ Psychiatry 2007; 190: 339-43.

32 Saha S, Scott JG, Johnston AK, Slade TN, Varghese D, Carter GL, et al. The association between delusional-like experiences and suicidal thoughts and behaviour. Schizophr Res 2011; 132: 197-202.
33 Kelleher I, Lynch F, Harley M, Molloy C, Roddy S, Fitzpatrick C, et al. Psychotic symptoms in adolescence index risk for suicidal behavior: findings from 2 population-based case-control clinical interview studies. Arch Gen Psychiatry 2012; 69: 1277-83. 